\title{
Outcomes of Mycobacterium avium complex lung disease based on clinical phenotype
}

\author{
Won-Jung Koh (1) ${ }^{1,7}$, Seong Mi Moon ${ }^{1,7}$, Su-Young Kim (10), Min-Ah Woo², \\ Seonwoo Kim², Byung Woo Jhun ${ }^{1}$, Hye Yun Park ${ }^{1}$, Kyeongman Jeon ${ }^{1}$, \\ Hee Jae Huh ${ }^{3}$, Chang-Seok Ki ${ }^{3}$, Nam Yong Lee ${ }^{3}$, Myung Jin Chung ${ }^{4}$, \\ Kyung Soo Lee ${ }^{4}$, Sung Jae Shin ${ }^{5}$, Charles L. Daley ${ }^{6}$, Hojoong Kim ${ }^{1}$ and 0 Jung Kwon ${ }^{1}$
}

Affiliations: ${ }^{1}$ Division of Pulmonary and Critical Care Medicine, Dept of Medicine, Samsung Medical Center, Sungkyunkwan University School of Medicine, Seoul, South Korea. ${ }^{2}$ Statistics and Data Center, Samsung Medical Center, Seoul, South Korea. ${ }^{3}$ Dept of Laboratory Medicine and Genetics, Samsung Medical Center, Sungkyunkwan University School of Medicine, Seoul, South Korea. ${ }^{4}$ Dept of Radiology, Samsung Medical Center, Sungkyunkwan University School of Medicine, Seoul, South Korea. ${ }^{5}$ Dept of Microbiology, Institute for Immunology and Immunological Diseases, Brain Korea 21 PLUS Project for Medical Science, Yonsei University College of Medicine, Seoul, South Korea. ${ }^{6}$ Division of Mycobacterial and Respiratory Infections, Dept of Medicine, National Jewish Health, Denver, CO, USA. ${ }^{7}$ Both authors contributed equally.

Correspondence: Won-Jung Koh, Division of Pulmonary and Critical Care Medicine, Dept of Medicine, Samsung Medical Center, Sungkyunkwan University School of Medicine, Irwon-ro 81, Gangnam-gu, Seoul 06351, South Korea. E-mail: wjkoh@skku.edu

@ERSpublications

Treatment outcomes differ according to clinical phenotype in patients with Mycobacterium avium complex lung disease http://ow.ly/g4WU30dbLHQ

Cite this article as: Koh W-J, Moon SM, Kim S-Y, et al. Outcomes of Mycobacterium avium complex lung disease based on clinical phenotype. Eur Respir J 2017; 50: 1602503 [https://doi.org/10.1183/ 13993003.02503-2016].

ABSTRACT The effect of the clinical phenotype of Mycobacterium avium complex (MAC) lung disease on treatment outcome and redevelopment of nontuberculous mycobacterial (NTM) lung disease after treatment completion has not been studied systematically.

We evaluated 481 treatment-naïve patients with MAC lung disease who underwent antibiotic treatment for $\geqslant 12$ months between January 2002 and December 2013.

Out of 481 patients, 278 (58\%) had noncavitary nodular bronchiectatic (NB) disease, 80 (17\%) had cavitary NB disease and 123 (25\%) had fibrocavitary disease. Favourable outcome was higher in patients with noncavitary disease $(88 \%)$ than in patients with cavitary disease $(76 \%$ for fibrocavitary and $78 \%$ for cavitary NB disease; $\mathrm{p}<0.05)$. Cavitary disease was independently associated with unfavourable outcomes $(\mathrm{p}<0.05)$. Out of 402 patients with favourable outcomes, 118 (29\%) experienced redevelopment of NTM lung disease, with the same MAC species recurring in 65 (55\%) patients. The NB form was an independent risk factor for redevelopment of NTM lung disease $(\mathrm{p}<0.05)$. In patients with recurrent MAC lung disease due to the same species, bacterial genotyping revealed that $74 \%$ of cases were attributable to reinfection and $26 \%$ to relapse.

Treatment outcomes and redevelopment of NTM lung disease after treatment completion differed by clinical phenotype of MAC lung disease.

This article has supplementary material available from erj.ersjournals.com

Received: Dec 202016 | Accepted after revision: June 242017

Support statement: This research was supported by the Basic Science Research Program through the National Research Foundation of Korea (NRF) funded by the Ministry of Science, ICT and future Planning (NRF-2015R1A2A1A01003959) and by a grant of the Korea Health Technology R\&D Project through the Korea Health Industry Development Institute (KHIDI), funded by the Ministry of Health \& Welfare, Republic of Korea (HI15C2778). The sponsor had no role in the design of the study, the collection and analysis of the data, or the preparation of the manuscript. Funding information for this article has been deposited with the Crossref Funder Registry.

Conflict of interest: Disclosures can be found alongside this article at erj.ersjournals.com

Copyright @ERS 2017 


\section{Introduction}

The incidence and prevalence of lung disease caused by nontuberculous mycobacteria (NTM) are increasing worldwide [1, 2]. Mycobacterium avium complex (MAC) predominantly consists of M. avium and $M$. intracellulare and is the most common aetiology of NTM lung disease worldwide [1,2]. MAC lung disease usually has two major clinical phenotypes: fibrocavitary and nodular bronchiectatic (NB) $[3,4]$. The fibrocavitary form is characterised by cavitary lesions that occur predominantly in the upper lobes and usually develops in older males with underlying lung disease, such as previous pulmonary tuberculosis and/or chronic obstructive pulmonary disease (COPD) [3]. The NB form occurs predominantly in postmenopausal, nonsmoking females [3] and can present as bilateral bronchiectasis with multiple nodules and tree-in-bud opacities on high-resolution computed tomography (HRCT). However, some patients with the NB form also have small cavitary lesions [5-7].

Macrolide-based combination antibiotic therapy is recommended for MAC lung disease, and current guidelines recommend different antibiotic regimens according to clinical phenotype: intermittent, three-times-weekly oral administration of three drugs for noncavitary NB (NC-NB) MAC lung disease; and daily oral drugs with or without parenteral drugs such as streptomycin or amikacin for cavitary MAC lung disease, including cavitary NB (C-NB) and fibrocavitary forms [3]. Treatment outcomes of the C-NB forms may be different from the NC-NB forms of MAC lung disease. However, many published studies that evaluated the clinical efficacy of macrolide-based antibiotic therapy did not differentiate between these clinical phenotypes [8-12], or included mainly NC-NB MAC lung disease and not cavitary disease [13-15].

In addition, recurrence of MAC lung disease or redevelopment of NTM lung disease is not uncommon after treatment completion [13, 16-18]. However, the impact of the clinical phenotype of MAC lung disease on treatment outcomes and on the recurrence of MAC lung disease or redevelopment of NTM lung disease has not yet been studied systematically. The purpose of the present study was to evaluate associations between clinical phenotypes and treatment outcomes, including recurrence of MAC lung disease or redevelopment of NTM lung disease after treatment completion, in patients with MAC lung disease.

\section{Methods \\ Study population}

Consecutive patients with MAC lung disease who received combination antibiotic treatment between January 2002 and December 2013 were identified using the database of the NTM registry of Samsung Medical Center (a 1979-bed referral hospital in Seoul, South Korea). Data from January 2002 to December 2007 were obtained from the retrospective cohort $[19,20]$, and data since January 2008 were obtained from an ongoing institutional review board-approved prospective observational cohort study to investigate NTM lung disease. Written informed consent was obtained from all participants (ClinicalTrials.gov identifier NCT00970801) [14].

The radiological phenotypes were classified according to the main features on chest radiography and HRCT. The fibrocavitary form was defined by the presence of cavitary opacities and pleural thickening, mainly in the upper lobes on chest radiograph and HRCT (online supplementary figure S1). The NB form was defined by the presence of multifocal bronchiectasis and clusters of small nodules on chest HRCT, regardless of the presence of small cavities in the lungs $[6,14]$. The NB form was further classified into cavitary (C-NB) versus noncavitary (NC-NB) according to the presence of cavitary lesion(s) (online supplementary figures S2 and S3). When the disease did not belong to either the fibrocavitary form or the NB form, such as for cases with solitary pulmonary nodules, it was deemed unclassifiable.

During a 12-year period, 714 patients with MAC lung disease started antibiotic treatment. All patients met the diagnostic criteria for NTM lung disease [3]. After excluding patients who had a history of previous treatment of NTM lung disease $(n=25)$, patients who had received prior antibiotic therapy for $>1$ month before transfer to our hospital $(n=82)$, and patients with an unclassifiable form $(n=41), 566$ patients were started on MAC therapy during the study period. 85 (15\%) patients were further excluded from the main analysis, because they received $<12$ months of antibiotic treatment due to discontinuation of antibiotics, loss to follow-up, transfer-out or death. The remaining 481 patients with newly diagnosed MAC lung disease, either the fibrocavitary or $\mathrm{NB}$ form, who received antibiotic therapy for $\geqslant 12$ months were included in the final study (figure 1).

\section{Microbiological examinations}

During the study period, MAC species were identified using PCR-restriction fragment length polymorphism analysis of the $r p o B$ gene or reverse-blot hybridisation of $r p o B[14,20]$. Drug susceptibility testing was performed using the broth microdilution method [21]. Isolates with a minimum inhibitory concentration of $\geqslant 32 \mu \mathrm{g} \cdot \mathrm{mL}^{-1}$ were considered resistant to clarithromycin [21]. For MAC isolates with 
Patients with MAC lung disease who started antibiotic treatment between January 2002 and December 2013 (n=714)

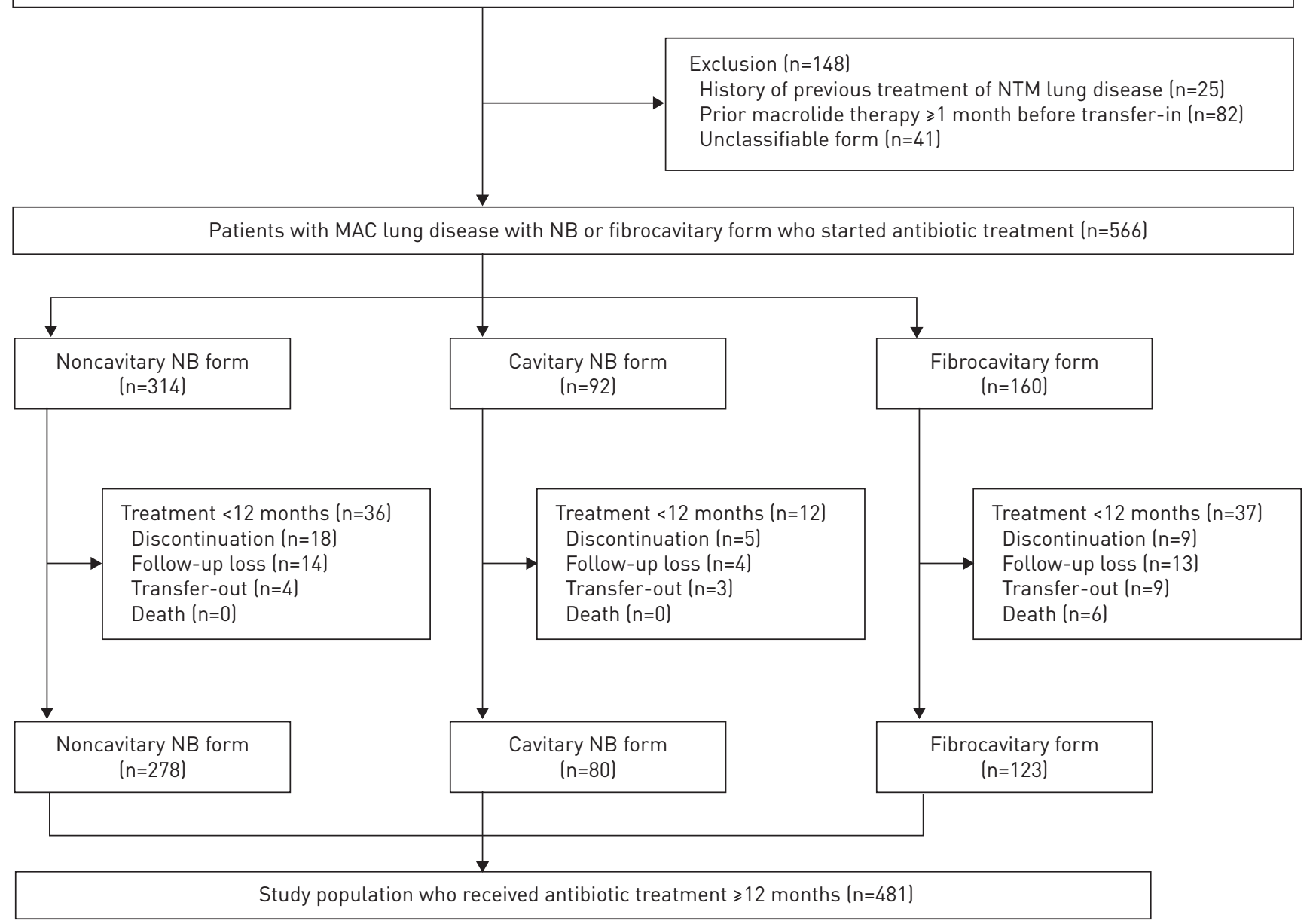

FIGURE 1 Study population. MAC: Mycobacterium avium complex; NTM: nontuberculous mycobacterial; NB: nodular bronchiectatic.

clarithromycin resistance, mutations in the $23 \mathrm{~S}$ rRNA gene were detected using PCR sequencing, as described previously [22].

In this study, "redevelopment" of NTM lung disease was defined as the subsequent diagnosis of NTM lung disease, regardless of causative NTM organism, after treatment completion of MAC lung disease. Cases where MAC lung disease had reappeared due to a different species were classed only as redevelopment. "Recurrence" of MAC lung disease was defined as two or more positive cultures of a MAC species after treatment completion for the same species, i.e. a redevelopment of M. avium (or M. intracellulare) lung disease after treatment completion of lung disease due to the same organism [13]. In cases of recurrent MAC lung disease, "relapse" with the same MAC genotype and "reinfection" with a new MAC genotype were distinguished. Mycobacterial genotyping was performed using repetitive sequence-based PCR (rep-PCR), which was standardised according to the DiversiLab Mycobacterium kit protocol [23]. Reports for rep-PCR were generated based on the Kullback-Leibler method, and isolates with identical profiles or $>97 \%$ similarity were regarded as indistinguishable $[24,25]$.

\section{Antibiotic therapy and treatment outcomes}

All patients who began antibiotic therapy received standardised combination antibiotic therapy, which consisted of an oral macrolide (clarithromycin or azithromycin), rifampicin and ethambutol [3]. Streptomycin was administered intramuscularly three times a week in patients with severe disease for the first several months, at the discretion of the attending physician. In our institution, all patients with MAC lung disease were treated with daily regimens before January 2011. After January 2011, all patients with the NC-NB form were initially treated with intermittent, three-times-weekly therapy regimens [14].

Sputum examinations were performed at 1,3 and 6 months after initiation of antibiotic treatment and then at 2-3-month intervals during treatment. Sputum culture conversion was defined as three consecutive 
negative cultures, and the time to culture conversion was defined as the date of the first negative culture [14]. Favourable outcome was defined as sputum culture conversion after initiation of treatment and maintenance of a negative culture for $\geqslant 12$ months on treatment. Unfavourable outcome was defined as no sputum culture conversion or by death. Some clinical data were included in previous studies [14, 19, 20]. After treatment completion, the patients were typically followed-up with sputum examinations every 6 months. Data on final treatment outcomes, follow-up information including redevelopment of NTM lung disease, and analyses of stored MAC isolates were collected in the current study.

\section{Statistical analyses}

All data are presented as n (\%) for categorical variables and median (interquartile range (IQR)) for continuous variables. Data categorised according to the three clinical phenotypes were compared using the Kruskal-Wallis test for continuous variables and Fisher's exact test for categorical variables. If there was a significant difference among the three groups, then post hoc analysis for multiple comparisons was performed with Tukey's test using ranks for continuous variables and Fisher's exact test using the permutation method for categorical variables.

To investigate potential independent factors associated with either unfavourable outcomes or redevelopment of NTM lung disease, multiple logistic regression analysis and multiple Cox regression with backward selection, respectively, were used. Variables including sex, age, body mass index (BMI), aetiological species, clinical phenotypes, sputum smear positivity, combined chronic pulmonary aspergillosis (CPA), COPD, previous lung resection history, combined use of injectable drugs and surgical resection were added to the regression models. p-values and confidence intervals were corrected using Bonferroni's method for multiple testing in multiple regression analysis. When estimating redevelopment of NTM lung disease, death was considered as a competing risk.

The Kaplan-Meier method was used to estimate the cumulative rates of redevelopment of NTM lung disease and the log-rank test was used to compare curves of the rates. A two-sided $\mathrm{p}<0.05$ was considered significant. All data sets were analysed using SAS version 9.4 (SAS Institute, Cary, NC, USA) and R3.2.3 (R Development Core Team, Vienna, Austria; www.R-project.org).

\section{Results}

\section{Baseline characteristics}

Out of the 481 patients, 278 (58\%) had the NC-NB form, 80 (17\%) had the C-NB form, and $123(25 \%)$ had the fibrocavitary form of MAC lung disease (table 1). None of the patients tested positive for HIV. $M$. avium was the more common aetiologic organism in patients with the NC-NB form, whereas $M$. intracellulare was more common in patients with the fibrocavitary form $(\mathrm{p}<0.001)$. No isolates were resistant to clarithromycin in drug susceptibility testing at the time of diagnosis of MAC lung disease.

\section{Treatment modalities and outcomes}

All patients with C-NB and fibrocavitary forms were prescribed three oral drugs daily. About half (51\%) of the patients with the NC-NB form received intermittent drug regimens. Streptomycin injection was used more frequently in patients with cavitary (68\% (138 out of 203$)$ ) than noncavitary disease (26\% (72 out of 278), $\mathrm{p}<0.001)$. Total antibiotic treatment duration was longer in patients with cavitary (24.0 (18.926.5) months) than noncavitary disease (19.7 (15.9-24.1) months; $\mathrm{p}<0.001)$ (table 2).

A favourable outcome was achieved in $84 \%$ (402 out of 481) of patients, and was higher for NC-NB disease $(88 \%$ (246 out of 278$)$ ) than for cavitary disease ( $76 \%$ (94 out of 123 ) with the fibrocavitary form and $78 \%$ (62 out of 80 ) with the C-NB form; $\mathrm{p}=0.006$ and $\mathrm{p}=0.037$, respectively) (table 2 ). Unfavourable outcomes occurred in 16\% (79 out of 481) patients, and 24 (30\%) had died by the end of January 2016.

\section{Risk factors for unfavourable outcomes}

Of the 481 patients, the final multiple logistic regression model revealed that male sex (adjusted (a)OR 1.80 , 95\% CI 1.07-3.02; $\mathrm{p}=0.027$ ) and cavitary disease, including both the C-NB (aOR 2.36, 95\% CI 1.24$4.52 ; \mathrm{p}=0.009$ ) and fibrocavitary form ( $\mathrm{aOR} 1.99,95 \% \mathrm{CI} 1.11-3.54 ; \mathrm{p}=0.020$ ) were independently associated with unfavourable outcomes (table 3). For 203 patients with cavitary disease, surgical resection was the only independent factor negatively associated with favourable outcome (aOR $0.13,95 \%$ CI $0.02-$ $0.98 ; \mathrm{p}=0.048)$.

\section{Redevelopment of NTM lung disease after treatment completion}

Of the 402 patients who completed treatment, 118 (29\%) redeveloped NTM lung disease during the median (IQR) follow-up period of 13.6 (4.8-28.3) months, which was the time between treatment completion and the last hospital visit or death. The median (IQR) number of sputum examinations after 
TABLE 1 Baseline characteristics of the study population

\begin{tabular}{|c|c|c|c|c|c|c|c|}
\hline & \multirow[t]{2}{*}{ Noncavitary NB } & \multirow[t]{2}{*}{ Cavitary NB } & \multirow[t]{2}{*}{ Fibrocavitary } & \multicolumn{4}{|c|}{ p-value } \\
\hline & & & & & $\begin{array}{l}\text { Non-cavitary } \\
\text { NB versus } \\
\text { fibrocavitary\# }\end{array}$ & $\begin{array}{c}\text { Cavitary NB } \\
\text { versus } \\
\text { fibrocavitary }\end{array}$ & $\begin{array}{c}\text { Noncavitary } \\
\text { NB versus } \\
\text { cavitary NB\# }\end{array}$ \\
\hline Subjects & $278(58)$ & $80(17)$ & $123(25)$ & & & & \\
\hline Male & 94 (34) & $21(26)$ & $81(66)$ & $<0.001$ & $<0.001$ & $<0.001$ & 0.392 \\
\hline Age years & $58(50-66)$ & $57(52-66)$ & $62(48-70)$ & 0.358 & & & \\
\hline BMI $\mathrm{kg} \cdot \mathrm{m}^{-2}$ & 20.6 (19.1-22.3) & $20.0(18.3-21.0)$ & 19.4 (17.8-21.2) & $<0.001$ & $<0.001$ & 0.914 & 0.003 \\
\hline Nonsmokers & $223(80)$ & $65(81)$ & 58 (47) & $<0.001$ & $<0.001$ & $<0.001$ & 0.999 \\
\hline Aetiologic organism & & & & $<0.001$ & $<0.001$ & 0.423 & 0.145 \\
\hline $\begin{array}{l}\text { Mycobacterium } \\
\text { avium }\end{array}$ & $167(60)$ & $39(49)$ & $49(40)$ & & & & \\
\hline $\begin{array}{l}\text { Mycobacterium } \\
\text { intracellulare }\end{array}$ & $111(40)$ & $41(51)$ & $74(60)$ & & & & \\
\hline \multicolumn{8}{|l|}{ Underlying diseases } \\
\hline History of TB & $88(32)$ & $33(41)$ & $78(63)$ & $<0.001$ & $<0.001$ & 0.006 & 0.260 \\
\hline COPD & $41(15)$ & 7 (9) & $30(24)$ & 0.009 & 0.045 & 0.010 & 0.357 \\
\hline CPA & $1(0)$ & $1(1)$ & $18(15)$ & $<0.001$ & $<0.001$ & 0.001 & 0.537 \\
\hline Lung cancer & $4(1)$ & $0(0)$ & $7(6)$ & 0.019 & 0.056 & 0.056 & 0.687 \\
\hline $\begin{array}{l}\text { Previous lung } \\
\text { resection }\end{array}$ & $14(5)$ & 2 (3) & $13(11)$ & 0.048 & 0.087 & 0.087 & 0.747 \\
\hline Diabetes mellitus & $21(8)$ & $6(8)$ & $14(11)$ & 0.459 & & & \\
\hline $\begin{array}{l}\text { Sputum smear } \\
\text { positivity }\end{array}$ & 104 (37) & $61(76)$ & 105 (85) & $<0.001$ & $<0.001$ & 0.264 & $<0.001$ \\
\hline CRP $\mathrm{mg} \cdot \mathrm{dL}^{-1}$ & $0.14(0.05-0.44)$ & $0.16(0.07-0.39)$ & $0.50(0.15-1.64)$ & $<0.001$ & $<0.001$ & 0.002 & 0.851 \\
\hline
\end{tabular}

Data are presented as $\mathrm{n}(\%)$ or median (interquartile range), unless otherwise stated. $\mathrm{n}=481$. NB: nodular bronchiectatic form of Mycobacterium avium complex lung disease; BMI: body mass index; TB: tuberculosis; COPD: chronic obstructive pulmonary disease; CPA: chronic pulmonary aspergillosis; CRP: C-reactive protein. " : post hoc analysis using permutation method for multiple comparisons.

treatment completion did not differ between patients with the fibrocavitary $(2(1-4))$ and NB (2 (1-4)) forms $(\mathrm{p}=0.429)$. In these 118 patients, MAC lung disease from the same species recurred in $65(55 \%)$ cases (31 cases of $M$. avium and 34 cases of $M$. intracellulare) and NTM lung disease from a different NTM species redeveloped in $53(45 \%)$ cases (table 4). Recurrence rates of MAC lung disease (i.e. only cases involving the same species) did not differ between patients with M. avium (31 (14\%) out of 219 patients with favourable outcomes) and $M$. intracellulare (34 (19\%) out of 183 patients; $\mathrm{p}=0.163$ ).

\section{Risk factors for redevelopment of NTM lung disease}

NTM lung disease redeveloped in 33\% (103 out of 308) of patients with the NB form and 16\% (15 out of 94) of patients with the fibrocavitary form $(\mathrm{p}=0.001)$. However, rates did not differ between patients with the C-NB $(31 \%, 19$ out of 62$)$ and NC-NB forms $(34 \%, 84$ out of $246 ; \mathrm{p}=0.602)$. The cumulative rate of redevelopment of NTM lung disease was higher in patients with the NB form, regardless of the presence of cavities, than in those with the fibrocavitary form $(\mathrm{p}=0.015$; figure 2$)$. These differences remained significant after adjustment using multiple Cox regression ( $\mathrm{p}=0.011$; table 5).

\section{Genotyping of paired isolates in recurrent MAC lung disease}

In the 65 patients $(31 \mathrm{M}$. avium and $34 \mathrm{M}$. intracellulare) with a recurrence of MAC lung disease, paired clinical isolates were available for genotyping in 27 (42\%) patients, including 12 (39\%) out of 31 patients with M. avium and 15 (44\%) out of 34 patients with M. intracellulare. According to the rep-PCR profiles, $74 \%$ (20 out of 27) of the isolates had genotypes different from those of the original isolates, suggesting reinfection, whereas $26 \%$ (seven out of 27 ) had genotypes identical to the initial genotype, suggesting relapse (table 6 and online supplementary figure S4). The proportion of reinfections was higher in patients with the NB form $(82 \%, 18$ out of 22$)$ than in those with the fibrocavitary form (40\%, two out of five) among patients with a recurrence and available paired isolates, although the difference was not statistically significant $(\mathrm{p}=0.091)$. The median (IQR) time interval between treatment completion and recurrence was shorter in patients with relapse (6.0 (4.8-8.5) months) than with reinfection (13.0 (6.0-23.7) months; $\mathrm{p}=0.040)$.

Drug susceptibility testing revealed that all reinfection isolates (20 patients) were susceptible to clarithromycin. However, clarithromycin resistance developed in two (29\%) out of seven patients with 
TABLE 2 Treatment modalities and outcomes of the study population

\begin{tabular}{|c|c|c|c|c|c|c|c|}
\hline & Noncavitary NB & Cavitary NB & Fibrocavitary & \multicolumn{4}{|c|}{ p-value } \\
\hline Subjects & $278(58)$ & $80(17)$ & $123(25)$ & & & & \\
\hline $\begin{array}{l}\text { Time interval } \\
\text { between diagnosis } \\
\text { and treatment } \\
\text { months }\end{array}$ & $7.0(2.2-23.2)$ & $5.8(1.5-16.6)$ & $1.5(0.5-5.4)$ & $<0.001$ & $<0.001$ & $<0.001$ & 0.242 \\
\hline Treatment regimen $\pi$ & & & & $<0.001$ & $<0.001$ & 0.999 & $<0.001$ \\
\hline Daily & 135 (49) & $80(100)$ & $123(100)$ & & & & \\
\hline Intermittent & $143(51)$ & $0(0)$ & $0(0)$ & & & & \\
\hline Streptomycin & $72(26)$ & $48(60)$ & 90 (73) & $<0.001$ & $<0.001$ & 0.135 & $<0.001$ \\
\hline Duration months & $3.0(2.3-5.1)$ & $3.2(2.8-5.7)$ & $4.0(3.0-6.2)$ & 0.004 & 0.003 & 0.096 & 0.677 \\
\hline Surgical resection $^{+}$ & $11(4)$ & $5(6)$ & $20(16)$ & 0.510 & & & \\
\hline Treatment outcomes & & & & 0.003 & 0.006 & 0.999 & 0.037 \\
\hline Favourable & 246 (88) & $62(78)$ & $94(76)$ & & & & \\
\hline Unfavourable & 32 (12) & $18(22)$ & $29(24)$ & & & & \\
\hline $\begin{array}{l}\text { Time to culture } \\
\text { conversion months }\end{array}$ & $1.2(0.9-2.8)$ & $2.0(0.9-4.1)$ & $3.2(1.4-6.1)$ & $<0.001$ & $<0.001$ & 0.144 & 0.028 \\
\hline $\begin{array}{l}\text { Time from culture } \\
\text { conversion to } \\
\text { treatment } \\
\text { completion months }\end{array}$ & $16.3(13.5-22.0)$ & $17.2(13.6-22.5)$ & $18.2(13.6-22.0)$ & 0.195 & & & \\
\hline
\end{tabular}

Data are presented as $\mathrm{n}(\%)$ or median (interquartile range), unless otherwise stated. $\mathrm{n}=481$. NB: nodular bronchiectatic form of Mycobacterium avium complex lung disease. " : post hoc analysis using permutation method for multiple comparisons; ${ }^{\text {? }}$ : initial treatment regimen consisted of three oral drugs: a macrolide (clarithromycin or azithromycin), rifampicin and ethambutol; ${ }^{+}$: sputum culture conversion rates after surgical resection: $89 \%(32 / 36)$ in total, $100 \%(20 / 20)$ for fibrocavitary form, $80 \%(4 / 5)$ for cavitary NB and $73 \%(8 / 11)$ for noncavitary NB.

relapsed MAC lung disease. One of the relapse isolates had a point mutation at position 2058 and the other at position 2059 of the $23 \mathrm{~S}$ rRNA gene.

\section{Discussion}

This study evaluated treatment outcomes and redevelopment of NTM lung disease, based on the clinical phenotype of MAC lung disease. Our study included nearly 500 patients with MAC lung disease who had not received prior treatment, and we found that treatment outcomes and redevelopment of NTM lung disease differed according to the clinical phenotype. Favourable outcomes were higher in patients with NC-NB MAC lung disease, compared with patients with the C-NB and fibrocavitary forms. Redevelopment of NTM lung disease was not uncommon in patients with NB MAC lung disease, including those with the C-NB form.

Current guidelines recommend intermittent antibiotic therapy for initial treatment of NC-NB MAC lung disease [3], and recent studies have reported high sputum culture conversion rates (74-86\%) in these patients [13-15]. The sputum culture conversion rate in the present study was high (88\%) in patients with NC-NB disease, although about half of our patients received daily therapy, because intermittent therapy was not introduced until 2011 in our institution. We previously showed that treatment outcomes did not differ between daily therapy and intermittent therapy in these patients [14]. Therefore, intermittent therapy is a reasonable option for patients with NC-NB MAC lung disease [3].

Daily antibiotic therapy is recommended for patients with cavitary MAC lung disease [3], and parenteral drugs should be considered for the first several months for extensive disease, especially the fibrocavitary form [3]. However, information regarding treatment outcomes in patients with fibrocavitary MAC lung disease is limited [8-12]. Our study included $>120$ patients with the fibrocavitary form. Although favourable outcomes $(76 \%)$ for the fibrocavitary form were lower than for the NC-NB form (88\%), our 
TABLE 3 Risk factors for unfavourable outcomes in the study population

\begin{tabular}{|c|c|c|c|c|c|c|}
\hline & \multirow[t]{2}{*}{ Favourable } & \multirow[t]{2}{*}{ Unfavourable } & \multicolumn{2}{|c|}{ Univariable analysis } & \multicolumn{2}{|c|}{ Multivariable analysis } \\
\hline & & & OR $(95 \% \mathrm{CI})$ & p-value & $\begin{array}{l}\text { Adjusted OR } \\
\text { (95\% CI) }\end{array}$ & p-value \\
\hline Subjects & $402(84)$ & $79(16)$ & & & & \\
\hline Male & 153 (38) & $43(54)$ & $1.94(1.20-3.16)$ & $<0.001$ & $1.80(1.07-3.02)$ & 0.027 \\
\hline Age years & $58(50-67)$ & $61(52-70)$ & $1.02(1.00-1.04)$ & 0.055 & & \\
\hline $\mathrm{BMI} \mathrm{kg} \cdot \mathrm{m}^{-2}$ & $20.2(18.8-21.9)$ & $19.5(17.8-21.7)$ & $0.97(0.89-1.05)$ & 0.440 & & \\
\hline $\begin{array}{l}\text { Mycobacterium } \\
\text { intracellulare }\end{array}$ & $183(46)$ & $43(54)$ & $1.43(0.88-2.32)$ & 0.148 & & \\
\hline $\begin{array}{l}\text { Sputum smear } \\
\text { positivity }\end{array}$ & $215(54)$ & $55(70)$ & $1.99(1.19-3.35)$ & 0.009 & & \\
\hline COPD & $64(16)$ & $14(18)$ & $1.14(0.60-2.15)$ & 0.691 & & \\
\hline Concurrent CPA & $14(4)$ & $6(8)$ & $2.28(0.85-6.12)$ & 0.103 & & \\
\hline $\begin{array}{l}\text { Previous lung } \\
\text { resection }\end{array}$ & $23(6)$ & $6(8)$ & $1.35(0.53-3.44)$ & 0.524 & & \\
\hline \multicolumn{7}{|l|}{ Type of disease } \\
\hline Noncavitary NB & $246(61)$ & $32(41)$ & 1.00 & Ref. & 1.00 & Ref. \\
\hline Cavitary NB & $62(15)$ & $18(23)$ & $2.23(1.07-4.65)$ & 0.014 & $2.36(1.24-4.52)$ & 0.009 \\
\hline Fibrocavitary & $94(23)$ & 29 (37) & $2.37(1.26-4.48)$ & 0.002 & $1.99(1.11-3.54)$ & 0.020 \\
\hline $\begin{array}{l}\text { Use of } \\
\text { streptomycin }\end{array}$ & $169(42)$ & $41(52)$ & $1.49(0.98-2.41)$ & 0.108 & & \\
\hline Surgical resection & $32(8)$ & $4(5)$ & $0.62(0.21-1.80)$ & 0.375 & & \\
\hline
\end{tabular}

Data are presented as $\mathrm{n}(\%)$ or as median (interquartile range), unless otherwise stated. $\mathrm{n}=481$. BMI: body mass index; COPD: chronic obstructive pulmonary disease; CPA: chronic pulmonary aspergillosis; NB: nodular bronchiectatic form of Mycobacterium avium complex lung disease.

current study showed that active application of injection drugs and combined surgical resection could result in reasonable treatment outcomes in this type of advanced MAC lung disease.

The proportion of patients with and clinical significance of the combination of cavitary lesions with NB MAC lung disease have not been well studied, although the proportion of patients with this combination is reported to range from $12 \%$ to $25 \%[5-7,14]$. In the original report of NB MAC lung disease, $24 \%$ (five out of 21) of patients had cavitary lesions at presentation, and an additional 15\% (three out of 21) of patients developed cavities during the course of the disease [26]. In the present study, cavitary lesions were detected in 80 (22\%) out of 358 patients with NB MAC lung disease. We found that favourable outcomes for C-NB disease were lower $(78 \%)$ than for NC-NB disease (88\%), but similar to that of fibrocavitary disease $(76 \%)$. These results suggest that careful evaluation of the presence of cavitary lesions using HRCT scans and differentiation between C-NB and NC-NB forms are important before initiating antibiotic treatment in patients with NB MAC lung disease. In addition, further clinical research is needed to evaluate the clinical

TABLE 4 Redevelopment of nontuberculous mycobacterial (NTM) lung disease in 118 patients after successful treatment of Mycobacterium avium complex (MAC) lung disease

\begin{tabular}{|c|c|c|c|}
\hline Initial disease & Subjects & Redevelopment of NTM lung disease & Subjects \\
\hline \multirow[t]{4}{*}{ M. avium } & 65 & M. avium & $31(48)$ \\
\hline & & M. intracellulare & $15(23)$ \\
\hline & & M. abscessus complex & $14(22)$ \\
\hline & & Others ${ }^{\#}$ & $5(7)$ \\
\hline \multirow[t]{5}{*}{ M. intracellulare } & 53 & M. intracellulare & $34(64)$ \\
\hline & & M. avium & $9(17)$ \\
\hline & & M. abscessus complex & $7(13)$ \\
\hline & & M. kansasii & $1(2)$ \\
\hline & & Others ${ }^{\#}$ & $2(4)$ \\
\hline
\end{tabular}

Data are presented as $\mathrm{n}$ or $\mathrm{n}(\%) .{ }^{*}$ : including mixed infections. 
FIGURE 2 Cumulative rate of redevelopment of nontuberculous mycobacterial (NTM) lung disease after treatment completion of Mycobacterium avium complex lung disease.

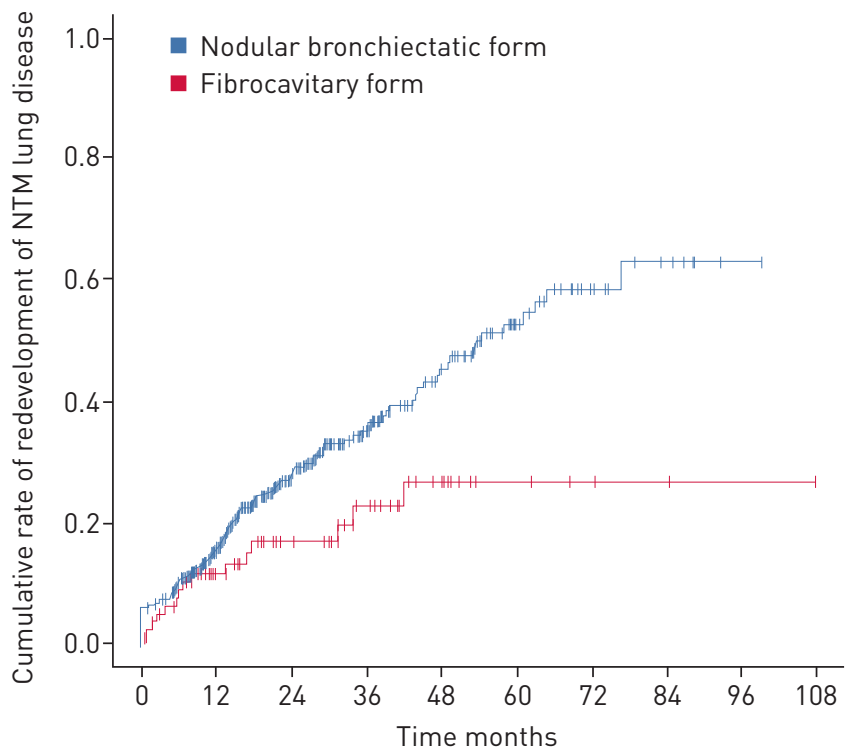

efficacy of optimal doses of macrolides [27-29], clofazimine [12, 30] and inhaled amikacin [31, 32] for cavitary MAC lung disease to improve treatment outcome.

Previous studies found that $22-50 \%$ of patients with MAC lung disease experience recurrence of MAC lung disease after successful treatment [13, 16-18]. Additionally, NTM lung disease caused by different species such as $M$. abscessus can develop during or after treatment of NB MAC lung disease [17, 33]. The present study revealed that lung disease due either to the same species of MAC or to a different NTM species could develop after treatment completion of MAC lung disease, and that this occurred more frequently with the C-NB and NC-NB forms than with the fibrocavitary form, consistent with previous reports [16]. Additionally, the majority of recurrences (i.e. from the same MAC species) were due to reinfection rather than true relapse. Patients with NB MAC lung disease were typically postmenopausal women who had a unique body morphotype, slender marfanoid body habitus (scoliosis, pectus excavatum and mitral valve prolapse), altered immunophenotype and mucociliary dysfunction [34-36], making them susceptible to environmental NTM exposure. Therefore, patients with the NB form should be followed-up, potentially for the duration of their lifetime, to detect and evaluate the recurrence of MAC or other NTM

TABLE 5 Risk factors for redevelopment of nontuberculous mycobacterial (NTM) lung disease after successful treatment of Mycobacterium avium complex (MAC) lung disease

\begin{tabular}{|c|c|c|c|c|c|c|}
\hline & \multirow[t]{2}{*}{ Redevelopment } & \multirow[t]{2}{*}{ No redevelopment } & \multicolumn{2}{|c|}{ Univariable analysis } & \multicolumn{2}{|c|}{ Multivariable analysis } \\
\hline & & & $\operatorname{HR}(95 \% \mathrm{CI})$ & p-value & Adjusted HR $(95 \% \mathrm{CI})$ & p-value \\
\hline Subjects & $118(29)$ & $284(71)$ & & & & \\
\hline Age years & $57(50-66)$ & $59(50-67)$ & $1.01(0.99-1.02)$ & 0.549 & & \\
\hline BMI $\mathbf{k g} \cdot \mathrm{m}^{-2}$ & 20.2 (18.9-21.5) & $20.2(18.8-22.1)$ & $1.00(0.94-1.06)$ & 0.976 & & \\
\hline Mycobacterium intracellulare & $53(45)$ & $130(46)$ & $1.01(0.70-1.45)$ & 0.953 & & \\
\hline CPA & $2(2)$ & $12(4)$ & $0.64(0.16-2.56)$ & 0.528 & & \\
\hline Previous lung resection & $6(5)$ & $17(6)$ & $0.88(0.38-2.02)$ & 0.761 & & \\
\hline \multicolumn{7}{|l|}{ Type of disease } \\
\hline Fibrocavitary form & $15(13)$ & $79(28)$ & 1.00 & Reference & 1.00 & Reference \\
\hline NB form & $103(87)^{\#}$ & $205(72)^{\pi}$ & $1.93(1.11-3.35)$ & 0.020 & $2.08(1.19-3.65)$ & 0.011 \\
\hline Use of streptomycin & $59(50)$ & 110 (39) & $0.88(0.61-1.26)$ & 0.490 & & \\
\hline
\end{tabular}




\begin{tabular}{|c|c|c|c|c|}
\hline & Total & Reinfection $\pi$ & Relapse ${ }^{\pi}$ & $\mathrm{p}$-value \\
\hline Subjects & 27 (100) & $20(74)$ & $7(26)$ & \\
\hline Type of disease & & & & 0.091 \\
\hline NB & $22(81)$ & $18(82)$ & $4(18)$ & \\
\hline Noncavitary NB & 17 & 14 & 3 & \\
\hline Cavitary NB & 5 & 4 & 1 & \\
\hline Fibrocavitary form & 5 (19) & $2(40)$ & $3(60)$ & \\
\hline Aetiology & & & & 0.091 \\
\hline M. avium & $12(44)$ & $11(92)$ & $1(8)$ & \\
\hline M. intracellulare & $15(56)$ & $9(60)$ & $6(40)$ & \\
\hline $\begin{array}{l}\text { Time interval between treatment } \\
\text { completion and recurrence months }\end{array}$ & $10.6(5.5-18.3)$ & $13.0(6.0-23.7)$ & $6.0(4.8-8.5)$ & 0.040 \\
\hline
\end{tabular}

Data are presented as $\mathrm{n}(\%), \mathrm{n}$ or median (interquartile range), unless otherwise stated. NB: nodular bronchiectatic form. \#: "recurrent MAC lung disease" only includes cases where MAC lung disease redeveloped due to the same species; " : repetitive sequence-based PCR profiles indicated that genotypes differed from the original isolates (reinfection) or were identical to the initial genotype (relapse).

lung disease. In contrast, genotyping results indicated that relapse developed more frequently in patients with the fibrocavitary form, suggesting that more effective drugs and longer treatment duration may be warranted with this form. Further studies regarding different treatment strategies against relapse and reinfection in MAC lung disease and methods to reduce environmental exposure in these patients are needed.

The present study has several limitations. First, this study was conducted at a single referral centre with specialised NTM clinics. Therefore, our results might not be generalisable to other centres and geographical areas. Second, patients receiving $<12$ months of antibiotic treatment (85 (15\%) out of 566) were excluded from the main analysis, and therefore favourable outcomes may have been overestimated; such patients were older, more likely to be male and more likely to have fibrocavitary disease (data not shown), and it is possible that older male patients with fibrocavitary disease might be less tolerant of daily antibiotic therapy with injectable drugs than patients with NC-NB disease who received intermittent antibiotic therapy. However, our results demonstrated that treatment outcomes are reasonably acceptable if patients receive treatment for $\geqslant 12$ months, which adheres to current guidelines. Third, administration of streptomycin was left to the discretion of the treating physician and not dependent on objective criteria. Fourth, there was no specific measure to monitor patients' compliance with treatment regimens. Fifth, paired clinical isolates were only available in $41 \%$ of patients with recurrent MAC lung disease. Sixth, because the follow-up period after successful treatment completion was relatively short, recurrence rates may have been underestimated. Seventh, there is potential overlap between the fibrocavitary and C-NB forms, because patients with the fibrocavitary form can have some nodular and bronchiectatic lesions in lower lung fields. Finally, we only differentiated between M. avium and M. intracellulare in our study, and several new species closely related to $M$. intracellulare, such as $M$. chimaera, were recently identified. Some studies suggest that recurrence rates differ between $M$. intracellulare and $M$. chimaera [37]. However, $M$. chimaera appears to be relatively rare in South Korea [38-40].

In conclusion, treatment outcome, including redevelopment of NTM lung disease and recurrence of MAC lung disease differed by clinical phenotype in patients with MAC lung disease. Favourable outcomes were lower in patients with cavitary MAC lung disease (both the fibrocavitary and C-NB forms). Redevelopment of NTM lung disease and recurrence of MAC lung disease were not uncommon in patients with NB MAC lung disease, even in those with the C-NB form. Therefore, different treatment regimens and possible interventions to prevent redevelopment or recurrence according to the clinical phenotype of MAC lung disease should be considered.

\section{Acknowledgements}

Author contributions are as follows. Study conception and design: W-J. Koh and S.M. Moon; data acquisition and analysis: W-J. Koh, S.M. Moon, M-A. Woo and S. Kim; experimental work: S-Y. Kim, H.J. Huh, Chang-Seok Ki, Nam Yong Lee and Sung Jae Shin; data interpretation and manuscript writing: Won-Jung Koh, Seong Mi Moon, Min-Ah Woo and S. Kim; critical revision and final approval of the manuscript: W-J. Koh, S.M. Moon, S-Y. Kim, M-A. Woo, S. Kim, B.W. Jhun, H.Y. Park, K. Jeon, H.J. Huh, C-S. Ki, N.Y. Lee, M.J. Chung, K.S. Lee, S.J. Shin, C.L. Daley, H. Kim and O.J. Kwon. 


\section{References}

1 Prevots DR, Marras TK. Epidemiology of human pulmonary infection with nontuberculous mycobacteria: a review. Clin Chest Med 2015; 36: 13-34

2 Stout JE, Koh WJ, Yew WW. Update on pulmonary disease due to non-tuberculous mycobacteria. Int J Infect Dis 2016; 45: 123-134.

3 Griffith DE, Aksamit T, Brown-Elliott BA, et al. An official ATS/IDSA statement: diagnosis, treatment, and prevention of nontuberculous mycobacterial diseases. Am J Respir Crit Care Med 2007; 175: 367-416.

4 Ryu YJ, Koh WJ, Daley CL. Diagnosis and treatment of nontuberculous mycobacterial lung disease: clinicians' perspectives. Tuberc Respir Dis 2016; 79: 74-84.

5 Kitada S, Uenami T, Yoshimura K, et al. Long-term radiographic outcome of nodular bronchiectatic Mycobacterium avium complex pulmonary disease. Int J Tuberc Lung Dis 2012; 16: 660-664.

6 Lee G, Lee KS, Moon JW, et al. Nodular bronchiectatic Mycobacterium avium complex pulmonary disease. Natural course on serial computed tomographic scans. Ann Am Thorac Soc 2013; 10: 299-306.

7 Gochi M, Takayanagi N, Kanauchi T, et al. Retrospective study of the predictors of mortality and radiographic deterioration in 782 patients with nodular/bronchiectatic Mycobacterium avium complex lung disease. BMJ Open 2015; 5: e008058.

8 Fujikane T, Fujiuchi S, Yamazaki Y, et al. Efficacy and outcomes of clarithromycin treatment for pulmonary MAC disease. Int J Tuberc Lung Dis 2005; 9: 1281-1287.

9 Lam PK, Griffith DE, Aksamit TR, et al. Factors related to response to intermittent treatment of Mycobacterium avium complex lung disease. Am J Respir Crit Care Med 2006; 173: 1283-1289.

10 Kobashi Y, Matsushima T. The microbiological and clinical effects of combined therapy according to guidelines on the treatment of pulmonary Mycobacterium avium complex disease in Japan - including a follow-up study Respiration 2007; 74: 394-400.

11 Miwa S, Shirai M, Toyoshima M, et al. Efficacy of clarithromycin and ethambutol for Mycobacterium avium complex pulmonary disease. A preliminary study. Ann Am Thorac Soc 2014; 11: 23-29.

12 Jarand J, Davis JP, Cowie RL, et al. Long-term follow-up of Mycobacterium avium complex lung disease in patients treated with regimens including clofazimine and/or rifampin. Chest 2016; 149: 1285-1293.

13 Wallace RJ Jr, Brown-Elliott BA, McNulty S, et al. Macrolide/azalide therapy for nodular/bronchiectatic Mycobacterium avium complex lung disease. Chest 2014; 146: 276-282.

14 Jeong BH, Jeon K, Park HY, et al. Intermittent antibiotic therapy for nodular bronchiectatic Mycobacterium avium complex lung disease. Am J Respir Crit Care Med 2015; 191: 96-103.

15 Griffith DE, Adjemian J, Brown-Elliott BA, et al. Semiquantitative culture analysis during therapy for Mycobacterium avium complex lung disease. Am J Respir Crit Care Med 2015; 192: 754-760.

16 Lee BY, Kim S, Hong Y, et al. Risk factors for recurrence after successful treatment of Mycobacterium avium complex lung disease. Antimicrob Agents Chemother 2015; 59: 2972-2977.

17 Min J, Park J, Lee YJ, et al. Determinants of recurrence after successful treatment of Mycobacterium avium complex lung disease. Int J Tuberc Lung Dis 2015; 19: 1239-1245.

18 Boyle DP, Zembower TR, Qi C. Relapse versus reinfection of Mycobacterium avium complex pulmonary disease. Patient characteristics and macrolide susceptibility. Ann Am Thorac Soc 2016; 13: 1956-1961.

19 Sim YS, Park HY, Jeon K, et al. Standardized combination antibiotic treatment of Mycobacterium avium complex lung disease. Yonsei Med J 2010; 51: 888-894.

20 Koh WJ, Jeong BH, Jeon K, et al. Clinical significance of the differentiation between Mycobacterium avium and Mycobacterium intracellulare in M. avium complex lung disease. Chest 2012; 142: 1482-1488.

21 Clinical Laboratory Standards Institute (CLSI). Susceptibility Testing of Mycobacteria, Nocardiae, and Other Aerobic Actinomycetes; Approved Standard. 2nd edn. CLSI document No. M24-A2. Clinical Laboratory Standards Institute, Wayne, PA, 2011.

22 Moon SM, Park HY, Kim SY, et al. Clinical characteristics, treatment outcomes, and resistance mutations associated with macrolide-resistant Mycobacterium avium complex lung disease. Antimicrob Agents Chemother 2016; 60: 6758-6765.

23 Healy M, Huong J, Bittner T, et al. Microbial DNA typing by automated repetitive-sequence-based PCR. J Clin Microbiol 2005; 43: 199-207.

24 Koh WJ, Jeong BH, Jeon $\mathrm{K}$, et al. Oral macrolide therapy following short-term combination antibiotic treatment for Mycobacterium massiliense lung disease. Chest 2016; 150: 1211-1221.

25 Koh WJ, Jeong BH, Kim SY, et al. Mycobacterial characteristics and treatment outcomes in Mycobacterium abscessus lung disease. Clin Infect Dis 2017; 64: 309-316.

26 Prince DS, Peterson DD, Steiner RM, et al. Infection with Mycobacterium avium complex in patients without predisposing conditions. N Engl J Med 1989; 321: 863-868.

27 van Ingen J, Egelund EF, Levin A, et al. The pharmacokinetics and pharmacodynamics of pulmonary Mycobacterium avium complex disease treatment. Am J Respir Crit Care Med 2012; 186: 559-565.

28 Koh WJ, Jeong BH, Jeon K, et al. Therapeutic drug monitoring in the treatment of Mycobacterium avium complex lung disease. Am J Respir Crit Care Med 2012; 186: 797-802.

29 Jeong $\mathrm{BH}$, Jeon K, Park HY, et al. Peak plasma concentration of azithromycin and treatment responses in Mycobacterium avium complex lung disease. Antimicrob Agents Chemother 2016; 60: 6076-6083.

30 Ferro BE, Meletiadis J, Wattenberg M, et al. Clofazimine prevents the regrowth of Mycobacterium abscessus and Mycobacterium avium type strains exposed to amikacin and clarithromycin. Antimicrob Agents Chemother 2016; 60: 1097-1105.

31 Olivier KN, Shaw PA, Glaser TS, et al. Inhaled amikacin for treatment of refractory pulmonary nontuberculous mycobacterial disease. Ann Am Thorac Soc 2014; 11: 30-35.

32 Olivier KN, Griffith DE, Eagle G, et al. Randomized trial of liposomal amikacin for inhalation in nontuberculous mycobacterial lung disease. Am J Respir Crit Care Med 2017; 195: 814-823.

33 Griffith DE, Philley JV, Brown-Elliott BA, et al. The significance of Mycobacterium abscessus subspecies abscessus isolation during Mycobacterium avium complex lung disease therapy. Chest 2015; 147: 1369-1375.

34 Kim RD, Greenberg DE, Ehrmantraut ME, et al. Pulmonary nontuberculous mycobacterial disease: prospective study of a distinct preexisting syndrome. Am J Respir Crit Care Med 2008; 178: 1066-1074. 
35 Kartalija M, Ovrutsky AR, Bryan CL, et al. Patients with nontuberculous mycobacterial lung disease exhibit unique body and immune phenotypes. Am J Respir Crit Care Med 2013; 187: 197-205.

36 Fowler CJ, Olivier KN, Leung JM, et al. Abnormal nasal nitric oxide production, ciliary beat frequency, and Toll-like receptor response in pulmonary nontuberculous mycobacterial disease epithelium. Am J Respir Crit Care Med 2013; 187: 1374-1381.

37 Boyle DP, Zembower TR, Reddy S, et al. Comparison of clinical features, virulence, and relapse among Mycobacterium avium complex species. Am J Respir Crit Care Med 2015; 191: 1310-1317.

38 Kim SY, Park HY, Jeong BH, et al. Molecular analysis of clinical isolates previously diagnosed as Mycobacterium intracellulare reveals incidental findings of "Mycobacterium indicus pranii" genotypes in human lung infection. BMC Infect Dis 2015; 15: 406.

39 Moon SM, Kim SY, Jhun BW, et al. Clinical characteristics and treatment outcomes of pulmonary disease caused by Mycobacterium chimaera. Diagn Microbiol Infect Dis 2016; 86: 382-384.

40 Kim SY, Shin SH, Moon SM, et al. Distribution and clinical significance of Mycobacterium avium complex species isolated from respiratory specimens. Diagn Microbiol Infect Dis 2017; 88: 125-137. 